Proceedings of the Institute of Mathematics and Mechanics,

National Academy of Sciences of Azerbaijan

Volume 46, Number 2, 2020, Pages 321-345

https://doi.org/10.29228/proc.35

\title{
ON SOME PROPERTIES OF LIMIT CYCLES OF THE BIRYUKOV EQUATION
}

\author{
YUSIF S. GASIMOV, SHARIF E. GUSEYNOV, AND JUAN E. NÁPOLES VALDÉS
}

\begin{abstract}
The present paper is the first of a series of works planned by the authors to study some qualitative properties of Liénard type equations solutions with derivatives of both natural and fractional order. In the present paper, we investigate the behaviour of limit cycles of the Biryukov equation, which is an important from the applicability standpoint special case of the classical Liénard equation.
\end{abstract}

\section{Introduction}

The classic Liénard equation concerning an unknown function $x=x(t) \not \equiv$ const is called the equation

$$
\ddot{x}+f(x) \dot{x}+g(x)=0,
$$

where $f \in C^{1}\left\{\mathbb{R}^{1}\right\}, g \in C^{1}\left\{\mathbb{R}^{1}\right\}$, at that $f(-x)=f(x)$ and $g(-x)=-g(x)$ for $\forall x \in \mathbb{R}^{1}$.

Let us consider (1.1) in the segment $\left[\alpha_{1}, \alpha_{2}\right]$, boundaries of which will be specified later for one particular case - for the Biryukov equation (looking ahead, we clarify that $\left.\alpha_{1}=-2 \sqrt{\frac{C}{L}}, \alpha_{2}=2 \sqrt{\frac{C}{L}}\right)$. Then, by the Weierstrass approximation theorem (for instance, see [55]), instead of the original equation (1.1), we can consider the equation

$$
\ddot{x}+F_{2 n}(x) \dot{x}+G_{2 m-1}(x)=0,
$$

where $F_{2 n}(x)$ and $G_{2 m-1}(x)$ are the polynomials of degree $2 n, n \in \mathbb{N} \cup\{0\}$ and $2 m-1, m \in \mathbb{N}$, respectively: $F_{2 n}(x)=\sum_{k=0}^{n} a_{2 k} x^{2 k}, G_{2 m-1}(x)=\sum_{k=1}^{m} b_{2 k-1} x^{2 k-1}$, at that $F_{2 n}^{(k)}(x) \stackrel{\text { unif. }}{\longrightarrow} f^{(k)}(x)$, and $G_{2 m-1}^{(k)}(x) \stackrel{\text { unif. }}{\longrightarrow} g^{(k)}(x)$ for $\forall x \in\left[\alpha_{1}, \alpha_{2}\right]$ and $\forall k=\{0 ; 1\}$.

The equation (1.2) is a general form for many equations describing some kinds of the linear or nonlinear oscillatory processes incipient in physics, biology, medicine and economics. For example:

2010 Mathematics Subject Classification. 34A34, 34C07.

Key words and phrases. Liénard equation, Biryukov equation, limit cycles, system oscillativity. 
- At $n=0, m=1, a_{0}=2 \alpha, b_{1}=\omega_{0}^{2}$ the equation (1.2) is the equation of shock-excited oscillation of the harmonic oscillator with a dissipation coefficient $\alpha$ (this coefficient having the dimension of frequency is often called the attenuation constant) and a proper frequency $\omega_{0}$ (for instance, see $[15])$ :

$$
\ddot{x}+2 \alpha \dot{x}+\omega_{0}^{2} x=0 .
$$

In spite of the fact that this equation describes the simplest oscillatory systems, it allows one to study such an important phenomenon as resonance - the effect of a sharp increase in the amplitude of oscillations when the frequency of action coincides with the natural frequency of the system.

- At $n=m=1, a_{0}=-\alpha, a_{2}=\alpha \beta, b_{1}=\omega_{0}^{2}$ the equation (1.2) presents the van der Pol tube generator equation, well known in radiophysics (for instance, see [15], [1]):

$$
\ddot{x}-\alpha\left(1-\beta x^{2}\right) \dot{x}+\omega_{0}^{2} x=0,
$$

where the parameter $\alpha>0$ shows how much the generator is excited; the parameter $\beta$ characterizes the amplitudes of the oscillations - as smaller is $\beta$, as greater is the amplitude. The van der Pol generator equation reveals a universal mechanism for the occurrence of both self-oscillations through Poincare-Andronov-Hopf bifurcations and quasiharmonic and relaxation oscillations (for instance, see [15], [1]). Note that the well-known Rayleigh equation (for instance, see [11], [18], [50], [52])

$$
\ddot{x}+\sigma\left(\dot{x}^{3}-\dot{x}\right)+x=0
$$

which occurs in hydrodynamics (for example, when studying the hydrodynamic stability of the parallel incompressible and inviscid shear flow) and in acoustics (for example, when studying statistical phenomena in the propagation of sound in the ocean; when studying the oscillations of a bubble in the field of acoustic pressure in a liquid, taking into account the compressibility of the gas inside the bubble; in the study of the formation and propagation of acoustic waves in an electromagnetic medium), after differentiation and replacement $\dot{x} \mapsto x$, is reduced to the equation of the van der Pol generator, in which $\alpha=\sigma, b=3, \omega_{0}=1$.

- At $n=0, m=2, a_{0}=\alpha, b_{1}=b_{3}=\omega_{0}^{2}$ the equation (1.2) turns to the Duffing equation (often called the Duffing oscillator; for instance, see [53], [49]):

$$
\ddot{x}+\alpha \dot{x}+\omega_{0}^{2}\left(1+x^{2}\right) x=0,
$$

which, despite being the simplest model of the oscillator with reactive nonlinearity, allows one to study the effects of nonlinear oscillatory systems such as nonisochronism, anharmonicity, and multistability.

- At $n=2, m=1, a_{0}=\lambda b, a_{2}=-\lambda c, a_{4}=\lambda d, b_{1}=\omega^{2}$ the equation (1.2) is the mathematical model for the colorectal myoelectrical activity in humans, offered in [37] in the form of the following highly excited oscillator, turning on at low values of the initial state and returning to the zero state - to its equilibrium point:

$$
\ddot{x}+\varepsilon\left(b-c x^{2}+d x^{4}\right) \dot{x}+\omega^{2} x=0,
$$


where $x$ characterizes the fluctuations of the transmembrane potential, the frequency of which is determined by the quantity $\omega$ and the constants $b, c, d$ are chosen so that the equation is characterized by the zero stable state and the unstable limit cycle, i.e. so that there are no oscillations, and the zero point, corresponding to the zero activity of the oscillator, is a stable point.

It should also be noted that some well-known non-linear equations or systems of equations (for example, system of coupled nonlinear oscillators of van der Pol, see [28], [3], [2]) describing various important from a practical point of view processes (for instance, see [4], [12], [17] and respective references given there), can be reduced to the equation of the form (1.1) (namely, to the equation of the form (1.1), and not to the Liénard equation (1.1), since the conditions $f(-x)=f(x)$, $g(-x)=-g(x)$ may not hold) and, therefore, to the equation of the form (1.2), if only the process under consideration allows one to consider (for example, if the process under consideration is periodic process: such process is, for example, bipedal locomotor, i.e. bipedal musculoskeletal system of human, animal or robot (see [4], [12] and respective references given there)). For example, the equation of motion for a relativistic harmonic oscillator

$$
\ddot{x}+\left(1-\dot{x}^{2}\right)^{\frac{3}{2}} x=0,
$$

that firstly was suggested in [4] (see also [39] and [13]), is reduced to the equation

$$
\ddot{x}+\left(1+3 x^{2} \sqrt{1-x^{2}}\right) \dot{x}+\left(1-x^{2}\right)^{\frac{3}{2}}=0,
$$

i.e. to the equation of the form (1.1) with the functions $f(x)=\left(1+3 x^{2} \sqrt{1-x^{2}}\right)$, $g(x)=\left(1-x^{2}\right)^{\frac{3}{2}}$ (it is obvious that $f(-x) \neq f(x), g(-x) \neq-g(x)$, therefore, the reduced equation is not a Liénard equation). In [39], the problem of finding the periodic solutions of the equation

$$
\frac{y}{\left(1-y^{2}\right)^{\frac{3}{2}}} d y+x d x=0
$$

is investigated. This equation characterizes the trajectories of the solutions of the original equation of motion for a relativistic harmonic oscillator in the phase space $(x, y=\dot{x})$ with "strip" structure: $\left\{(x, y): x \in \mathbb{R}^{1},|y|<1\right\}$. In [13], an interesting approximate iterative algorithm, first proposed in [10], which, in our opinion, is very promising, is applied to the original equation rather than to the reduced equation of the form (1.1) motion for the relativistic harmonic oscillator approach in the sense that it can be modified for the "peculiar demand" of many of the higher order nonlinear ordinary differential equations.

In the general case, the equation (1.1) cannot be solved by the analytical methods: even for most of the above equations, which are not the most complicated particular cases of the equation (1.1), no analytical solutions were found. Currently, there are many numerical methods for solving nonlinear equations and systems of equations, in particular, equations of the form (1.1) and (1.2) (for instance, see [8], [7], [33], [23], [24]; see also the fundamental textbooks [26] and [47] on the theory and applications of nonlinear ordinary differential equations), which have significantly different accuracy, stability, convergence rate, complexity. As 
shown in [45], questions of accuracy, stability, and complexity when choosing one or another numerical method are especially relevant when conducting numerical analysis of the self-oscillating circuits in the time domain: equations and systems of equations describing such schemes are almost always simultaneously are oscillating and rigid, and, therefore, the effectiveness of the selected numerical method for solving such equations requires additional research. Despite the fact that there are many software packages for solving rigid and non-rigid systems of ordinary differential equations (for instance, see [8], [7], [33], [23], [24]), each of which contains one or more schemes and strategies for choosing a step and, most likely, strategies for choosing the scheme itself, reliable theoretical comparison of these packages is very difficult, since reliable theoretical comparison should take into account the order of accuracy, A- and L-stability (for instance, see [24]), complexity, etc. but high order requirements and, good stability and a small amount of calculations for the same schemes often contradict each other, and a priori impossible to say which is better - a high order of accuracy or a good resistance. A theoretical comparison of step selection strategies is even less obvious.

In the next two sections, some properties of limit cycles of the Biryukov equation is investigated. The Biryukov equation is a special case of the classical Liénard equation having inportant significance from the standpoint of its application in the self-oscillating circuits' systems in the time domain.

\section{The Biryukov equation (Biryukov oscillator)}

One of the special cases of the Liénard equation (1.1) is the Biryukov equation [5]), sometimes called the Biryukov oscillator, or simply the LC-oscillator (inductance-capacitance oscillator), which describes the auto-oscillations and used to model damped oscillators:

$$
\ddot{x}+\frac{1}{C} \frac{d R}{d x} \dot{x}+\frac{1}{L C} x=0,
$$

where the function $x=x(t)$ characterizes the voltage on the capacitance of the oscillator; by $C=$ const $>0$ is denoted the indicated linear capacitance of the oscillator; $L=$ const $>0$ denotes the linear inductance of the oscillator; the function $R=R(x)$ describes the current-voltage characteristic of the nonlinear resistive element of the oscillator, and it is required fulfillment of the condition

$$
2 R(0)=R(x)+R(-x) .
$$

The Biryukov equation (2.1) directly follows from the system of equations of self-oscillation for the variable states of the oscillator (for instance, see [46]):

$$
\left\{\begin{array}{l}
-C \dot{x}=R+I_{\text {current }}, \\
L \dot{I}_{\text {current }}=x
\end{array}\right.
$$

where the function $I_{\text {current }}=I_{\text {current }}(t)$ characterizes the inductance current.

Note that the equation (2.1) is not a special case of the Liénard equation (1.1) if the condition (2.2) is not satisfied.

If in the equation $(2.1)$ the conductivity function $\frac{d R}{d x}$ takes a negative value at any segment of the current-voltage characteristic of the nonlinear resistive 
element of the oscillator, it is quite possible that self-oscillations will occur in the LC circuit of the oscillator.

If in the equation (2.1) approximate the current-voltage characteristic of the nonlinear resistive element of the oscillator by the third power polynomial $R(x)=$ $\sum_{k=0}^{3} a_{k} x^{k}$, where $a_{0}=R(0)$ and $a_{2}=0$, then the equation (2.1) will turn to the van der Pol equation, the analytical solution of which cannot be obtained in explicit form (only for the steady-state regime its analytical solutions are known, see [13]), and only the educational-benchmark numerical solutions specially calculated with high accuracy for some parameters of the equation (2.1) and the initial data (see $[5],[19])$, and these training-reference numerical solutions were found at certain points, which does not allow the acceptable calculation of the error rates. It should be noted that even an exact solution of the equation (2.1) with a cubic approximation of the function $R=R(x)$ would not have any practical value, since the accuracy of the approximation itself is insufficient.

In the work of [45], a rather deep comparative analysis of several basic modern numerical methods was carried out (their effectiveness was also evaluated) to solve the Biryukov equation (2.1) with piecewise-linear approximation of the currentvoltage characteristic of the nonlinear resistive element of the oscillator: the obtained simple model allows firstly, to reduce the approximation error of the current-voltage characteristics, and, secondly, to obtain the exact solution for the stationary mode, which is the most important mode for practice (see [5]). Below we will study some properties of the limit cycles of the equation (2.1) regardless of the fulfillment or non-fulfillment of the condition (2.2) (as mentioned above, in the Biryukov equation (2.1), the condition (2.2) is mandatory; if the condition (2.2) is not satisfied, then the equation (2.1), which in itself is an important equation from the point of view of application, ceases to be a particular case of the Liénard equation and, therefore, is not the Biryukov equation).

\section{Behavior of the limit cycles of the Biryukov equation}

In this section, some properties of limit cycles of the Biryukov equation (3) will be studying. For this purpose, in the equation (2.1) we pass to the polar coordinate system introducing the designations $\dot{x}=r \sin (\varphi)$, where $r=r(t)>0$, $\varphi=\varphi(t) \in[0,2 \pi)$ (or, it is the same, $\varphi=\varphi(t) \in(-\pi, \pi]$; for instance, see [51]). Then instead of the equation (2.1) we get the following system:

$$
\left\{\begin{array}{l}
\cos (\varphi) \dot{r}-r \sin (\varphi) \dot{\varphi}=r \sin (\varphi), \\
\sin (\varphi) \dot{r}+r \cos (\varphi) \dot{\varphi}=\frac{1}{C}\left(\frac{\partial R(r \cos (\varphi))}{\partial \varphi}\right. \\
\left.-r \tan (\varphi) \frac{\partial R(r \cos (\varphi))}{\partial r}-\frac{1}{L} r \cos (\varphi)\right),
\end{array}\right.
$$

from which it is easy to find expressions for $\dot{r}$ and $\dot{\varphi}$ :

$$
\begin{aligned}
& \dot{r}=\frac{1}{L C}\left(L \frac{1}{r} \frac{\partial R(r \cos (\varphi))}{\partial \varphi}-L \tan (\varphi) \frac{\partial R(r \cos (\varphi))}{\partial r}\right. \\
& -(1-L C) \cos (\varphi)) r \sin (\varphi)
\end{aligned}
$$




$$
\begin{aligned}
& \dot{\varphi}=\frac{1}{L C}\left(L \cos (\varphi) \frac{1}{r} \frac{\partial R(r \cos (\varphi))}{\partial \varphi}-L \sin (\varphi) \frac{\partial R(r \cos (\varphi))}{\partial r}\right. \\
& \left.+(1-L C) \sin ^{2}(\varphi)-1\right) .
\end{aligned}
$$

The obtained expressions allow us to rewrite the equation (2.1) in the following equivalent form:

where

$$
\frac{d r}{d \varphi}=\frac{r P_{1}(r, \varphi)}{P_{2}(r, \varphi)}
$$

$$
\begin{aligned}
& P_{1}(r, \varphi)=\left(L \tan (\varphi) \frac{\partial R(r \cos (\varphi))}{\partial r}+(1-L C) \cos (\varphi)\right) r \\
& -L \frac{\partial R(r \cos (\varphi))}{\partial \varphi}, \\
& P_{2}(r, \varphi)=\left(L \frac{\partial R(r \cos (\varphi))}{\partial r}-(1-L C) \sin (\varphi)+\frac{1}{\sin (\varphi)}\right) r \\
& -L \cot (\varphi) \frac{\partial R(r \cos (\varphi))}{\partial \varphi} .
\end{aligned}
$$

The functions $P_{1}(r, \varphi)$ and $P_{2}(r, \varphi)$ have the following representations:

$$
\begin{aligned}
& P_{1}(r, \varphi)=A(r, \varphi) \sin (\varphi)+B(r, \varphi) \cos (\varphi), \\
& P_{2}(r, \varphi)=A(r, \varphi) \cos (\varphi)-B(r, \varphi) \sin (\varphi),
\end{aligned}
$$

in which the essence of the functions $A(r, \varphi)$ and $B(r, \varphi)$ is quite obvious:

$$
\begin{gathered}
A(r, \varphi)=\left.\left.A(x, \dot{x})\right|_{x=r \cos (\varphi) ; \dot{x}=r \sin (\varphi)} \stackrel{\text { def }}{\equiv}\left(L \frac{d R(x)}{d x} \dot{x}+x\right)\right|_{x=r \cos (\varphi) ; \dot{x}=r \sin (\varphi)}, \\
B(r, \varphi)=\left.A(x, \dot{x})\right|_{x=r \cos (\varphi) ; \dot{x}=r \sin (\varphi)} \stackrel{\text { def }}{\equiv}-\left.L C \dot{x}\right|_{\dot{x}=r \sin (\varphi)} .
\end{gathered}
$$

If we assume that the equation (3.1) has a closed periodic trajectory (limit cycle) containing the origin, then it is possible (its occurrence depends on the concrete expression of the function $R(r, \varphi)$ ) that the solution $r(\varphi)$ of the equation (3.1) will be a single-valued function of the variable $\varphi$, for which $\frac{d r}{d \varphi}$ does not tend to infinity for any value of the argument $\varphi$. Then the solution $r(\varphi)$ will be a periodic function of the period $2 \pi$, and therefore, we can write:

$$
r(\varphi)=\frac{\alpha_{0}}{2}+\sum_{k=1}^{+\infty}\left(\alpha_{k} \cos (k \varphi)+\beta_{k} \sin (k \varphi)\right),
$$

where $\left\{\alpha_{k}\right\}_{k \in \mathbb{N} \cup\{0\}}$ and $\left\{\beta_{k}\right\}_{k \in \mathbb{N}}$ are the Fourier coefficients, or in the equivalent form:

$$
r(\varphi)=\sum_{k=-\infty}^{+\infty} r_{k} e^{i k \varphi}
$$

where $i$ is an imaginary unit; $r_{0}=\frac{\alpha_{0}}{2} ; r_{k}=\frac{\alpha_{|k|}-\operatorname{sgn}(k) \beta_{|k|} i}{2}, k \in \mathbb{Z} \backslash\{0\}$.

Introducing the notation $y=e^{i \varphi}$, we obtain that the denominator and numerator of the right hand side of the equation (3.1) are polynomials with respect to variables $r$ and $y$ (more precisely, they are power series that we can cut off with any desired accuracy - the conditions we are considering allow this to be done), 
and the solution (3.2) which now takes the form (as the Laurent series about the origin)

$$
r(y)=\sum_{k=-\infty}^{+\infty} r_{k} y^{k},
$$

is a single-valued function that can have essential singularity at points $y=0$ and $y=\infty$. Then, by virtue of the fact (for instance, see [16]), that any single-valued integral of the differential equation

$$
\frac{d r}{d y}=\frac{\tilde{P}_{1}(r, y)}{\tilde{P}_{2}(r, y)},
$$

where $\tilde{P}_{1}(r, y)$ and $\tilde{P}_{2}(r, y)$ are polynomials, $r(y)$ is a rational function, unless the differential equation under consideration is a Riccati equation. This means that on the right-hand side of (3.3) only a finite number of coefficients are nonzero and, moreover, the solution of (3.3) takes the following form: $r(y)=\frac{Q_{2 N}(y)}{y^{N}}$, where $N$ is some integer. Therefore, having performed the inverse transformation $(r, y) \mapsto(r, \varphi)$, we obtain a trigonometric polynomial $r(\varphi)=\sum_{k=0}^{N} r_{k} e^{i k \varphi}$, i.e. we obtained that the closed trajectory of the equation (3.1) (or the original equation (2.1), what is the same) is an algebraic curve if only the function $R(r, \varphi)$ (or the function $R(x)$ in the original equation (2.1)) is such that

$$
P_{1}(r, \varphi) \neq\left(U_{1}(\varphi) r+U_{2}(\varphi) \frac{1}{r}+U_{3}(\varphi)\right) P_{2}(r, \varphi)
$$

where $U_{j}(\varphi), j=\overline{1,3}$ are some functions of the argument $\varphi$.

Investigation of the case, when

$$
P_{1}(r, \varphi)=\left(U_{1}(\varphi) r+U_{2}(\varphi) \frac{1}{r}+U_{3}(\varphi)\right) P_{2}(r, \varphi),
$$

i.e. when the equation is a Riccati equation, will not be carried out in this paper, however, it should be noted that if the case (3.4) holds, then either all solutions of the equation (3.1) are periodic or the number of periodic solutions, if any, is at most two (in other words, either all solutions of the equation (2.1) are closed or the equation (2.1) can have no more than two limit cycles); and if the equation (3.1) has at least one periodic solution, then the original equation (2.1) has only one singular point. It is appropriate to note here that in the fundamental work [35] (see short notes [44], [36], in which the matter is the made some errors and the ways to correct them) it was proved that the number of limit cycle of the equation

$$
\dot{x}=\frac{P(x, t)}{Q(x, t)},
$$

where $P(x, t)$ and $Q(x, t)$ are polynomials of degree no higher than $n$, does not exceed the number

$$
\frac{6 n^{3}-7 n^{2}+g(n)}{2}
$$


where

$$
g(n)=\left\{\begin{array}{l}
16-11 n \text { if } n \text { is an odd integer, } \\
n+4 \text { if } n \text { is an even integer. }
\end{array}\right.
$$

It should be noted that the majorant estimate (3.6) is too rough (exaggerated) estimate. The truth of the above follows, for example, from the well-known fact (see [43]) that one can construct an equation of the form (3.5), which is asymptotically close to the differential equation of one-parameter family of concentric circles

$$
x d t+t d t=0
$$

and which has exactly $\frac{n^{2}+5 n-14}{2}, n \geq 3$ limit cycles (i.e. there is an equation of the form (3.5), having: for $n=3$ exactly 5 limit cycles, for $n=4$ exactly 11 limit cycles, for $n=5$ exactly 18 limit cycles, and so on).

The truth of this remark also follows from the fact proved in the underlying paper [34] (using the results of this article should also be taken into account the short notes [44], [36]) that, if $P(x, t)$ and $Q(x, t)$ are polynomials of the second degree, then the equation (3.5) cannot have more than three limit cycles.

The Fig. 1 contains a printout of a program in Mathcad, version 14.0.0.163, that implements the formula (3.6) for $n=\overline{1,20}$. As can be seen from the Fig. 1, when $n=2$, i.e. when $P(x, t)$ and $Q(x, t)$ are polynomials of the second degree, the upper bound (3.6) of the number of possible limit cycles of the equation (3.5) is 13 (which is more than 4 times greater than the permissible value of 3 ).

Finally, we note that if in the equation (3.5) the condition

$$
P(x, t) \neq\left(U_{1}(t) x^{2}+U_{2}(t) x+U_{3}(t)\right) Q(x, t)
$$

is satisfied, where $U_{j}(t), j=\overline{1,3}$ are some functions of the argument $t$, and if its reduced equation

where

$$
\frac{d r}{d \varphi}=\frac{r \tilde{P}(r, \varphi)}{\tilde{Q}(r, \varphi)}
$$

$$
\begin{aligned}
& \tilde{P}(r, \varphi)=P(r \cos (\varphi), r \sin (\varphi)) \sin (\varphi)+Q(r \cos (\varphi), r \sin (\varphi)) \cos (\varphi), \\
& \tilde{Q}(r, \varphi)=P(r \cos (\varphi), r \sin (\varphi)) \cos (\varphi)-Q(r \cos (\varphi), r \sin (\varphi)) \sin (\varphi),
\end{aligned}
$$

takes place the representation

$$
\frac{\tilde{P}(r, \varphi)}{\tilde{Q}(r, \varphi)}=\tilde{U}_{1}(\varphi) r+\tilde{U}_{2}(\varphi) \frac{1}{r}+\tilde{U}_{3}(\varphi),
$$

where $\tilde{U}_{j}(\varphi), j=\overline{1,3}$ are some functions of the argument $\varphi$, then the equation (3.5) can have a countable number of periodic solutions that are not trigonometric polynomials (but are reduced to them) (for instance, see [30], [25]). For example, the next two equations:

and

$$
\dot{x}=\frac{P_{e x .1}(x, t)}{Q_{e x .1}(x, t)}
$$

where

$$
\dot{x}=\frac{P_{e x .2}(x, t)}{Q_{e x .2}(x, t)},
$$

$$
P_{e x .1}(x, t)=x^{3}-t x^{2}-t^{2} x-t^{3},
$$




$$
\begin{aligned}
& P_{\text {ex. } 2}(x, t)=(1+t) x^{2}+t x+t^{3}, \\
& Q_{\text {ex.1 }}(x, t)=x^{3}-t x^{2}+t^{2} x-t^{3}, \\
& Q_{\text {ex. } 2}(x, t)=x^{3}+t(1-t) x+t^{2},
\end{aligned}
$$

for which the condition (3.7) is satisfied, have the following reduced equations, respectively:

$$
\begin{gathered}
\frac{d r}{d \varphi}=r \cos (2 \varphi), \\
\frac{d r}{d \varphi}=\cos (\varphi)+\sin (\varphi),
\end{gathered}
$$

for which, obviously, (3.8) holds. The integral curves of the both equations are shown in Fig. 2 and Fig. 3, respectively (implemented on Mathcad, version 14.0.0.163).

Now we consider the equation (2.1) taking into account the condition (2.2), i.e. we consider the Biryukov equation, which, as already mentioned above, is a special case of the Liénard equation. Above, starting from the place, where the transition to the polar coordinate system was made in the equation (2.1), we repeatedly proceeded from the fact that current-voltage characteristic of nonlinear resistive element of the damped oscillator (2.1) possesses the properties we need - mainly those or other quantitative properties (at the same time, it was not required to satisfy the condition $(2.2))$.

Now, under the assumption that the condition (2.2) is satisfied in the equation (2.1), we will try to find simple qualitative conditions for the conductivity function $\frac{d R}{d x}$, the fulfillment of which ensures the uniqueness of the solution of the Biryukov equation (2.1). To do this, we consider the equation of the curve $P_{2}(r, \varphi)=0$, whose left-hand side is the denominator of the right-hand side of the equation (3.1), which is the reduced equation for the original equation (2.1).

The curve $P_{2}(r, \varphi)=0$ (more precisely, the curve $\tilde{P}_{2}(r, \varphi)=0$, where $\left.\tilde{P}_{2}(r, \varphi)=\frac{1}{r} \sin (\varphi) P_{2}(r, \varphi)\right)$ can be interpreted as the intersection of the surfaces $z_{1}(r, \varphi)$ and $z_{2}(r, \varphi)$, given by the formulas

$$
\begin{gathered}
z_{1}(r, \varphi)=\frac{L}{\cos (\varphi)} \frac{\partial R(r \cos (\varphi))}{\partial r}-\frac{L}{r \sin (\varphi)} \frac{\partial R(r \cos (\varphi))}{\partial \varphi} \\
z_{2}(r, \varphi)=(1-L C) \tan (\varphi)-\frac{2}{\sin (2 \varphi)}
\end{gathered}
$$

Thus, let us consider the curve

$$
\begin{aligned}
& \tilde{P}_{2}(r, \varphi) \stackrel{\text { def }}{=} L \sin (\varphi) \frac{\partial R(r \cos (\varphi))}{\partial r}-L \cos (\varphi) \frac{1}{r} \frac{\partial R(r \cos (\varphi))}{\partial \varphi} \\
& -(1-L C) \sin ^{2}(\varphi)+1=0 .
\end{aligned}
$$

First of all, we note that from the equation (3.9) it immediately follows that $\varphi \neq \frac{\pi k}{2}, k \in \mathbb{Z}$ (since in this work we assume $\varphi \in[0,2 \pi)$, fulfilment of the condition $\varphi \neq \frac{\pi k}{2}, k=\overline{0,3}$ is enough for us). We will call this the first fact. In view of this fact, we will try to find conditions under which on each connected component of the curve (3.9) the mapping $r=r(\varphi):[0,2 \pi) \backslash\left\{\frac{\pi k}{2}, k=\overline{0,3}\right\} \rightarrow$ $(0,+\infty)$ is defined as a single-valued function of variable $\varphi$. 
Multiplying (3.9) by $r^{2}$ and making replacement $x=r \cos (\varphi)$ in the obtained expression, we come to the quadratic equation

$$
L C y^{2}+\left(L x \frac{d R(x)}{d x}\right) y+x^{2}=0,
$$

existence of real solution of which is ensured by the condition

$$
\frac{d R(x)}{d x} \in\left(-\infty,-2 \sqrt{\frac{C}{L}}\right] \cup\left[2 \sqrt{\frac{C}{L}},+\infty\right),
$$

ensuing from non-negativity of the discriminant of the equation (3.10). We will call this the second fact.

Obviously, at $\frac{d R(x)}{d x} \in\left(-2 \sqrt{\frac{C}{L}}, 2 \sqrt{\frac{C}{L}}\right)$ the equation (3.10) has no real roots and, therefore, the curve (3.9) has no connected components. From this, taking into account $\dot{\varphi}=-\frac{1}{L C} \tilde{P}_{2}(r, \varphi)$, it follows that $\dot{\varphi}$ preserves its sign, i.e. $\varphi=\varphi(t)$ is a strictly monotone function in the case of $\frac{d R(x)}{d x} \in\left(-2 \sqrt{\frac{C}{L}}, 2 \sqrt{\frac{C}{L}}\right)$.

To reason in a similar way, it is easy to verify that in this case $\dot{r}$ also preserves its sign (it has a negative sign for $\varphi \in\left(\pi k, \frac{\pi}{2}+\pi k\right), k=\{0 ; 1\}$ and has a positive sign for $\left.\varphi \in\left(-\frac{\pi}{2}+\pi k, \pi k\right), k=\{1 ; 2\}\right)$.

From the condition (3.11) follows that the function $R(x)$, which is the currentvoltage characteristic of the nonlinear resistive element of the damped oscillator (2.1), should be a strictly monotonic function outside the interval $\left(-2 \sqrt{\frac{C}{L}}, 2 \sqrt{\frac{C}{L}}\right)$ once $R(x)$ is considered in $\mathbb{R}^{1}$, namely, $R(x)$ strictly increases on the infinite right half-interval $\left[2 \sqrt{\frac{C}{L}},+\infty\right)$ and strictly decreases on the infinite left half-interval $\left(-\infty,-2 \sqrt{\frac{C}{L}}\right]$.

Thus, taking into account the above two facts, we can state that for the connected components of the curve (3.9) we have the inequality

$$
\left[\begin{array}{l}
z_{1}(r, \varphi) \geq 2 \sqrt{\frac{C}{L^{3}}},(r ; \varphi)=\left(r>0 ; \varphi \in[0,2 \pi) \backslash\left\{\frac{\pi k}{2}, k=\overline{0,3}\right\}\right), \\
z_{1}(r, \varphi) \leq-2 \sqrt{\frac{C}{L^{3}}},(r ; \varphi)=\left(r>0 ; \varphi \in[0,2 \pi) \backslash\left\{\frac{\pi k}{2}, k=\overline{0,3}\right\}\right) .
\end{array}\right.
$$

Now, let us assume $\exists \varphi^{*} \in[0,2 \pi) \backslash\left\{\frac{\pi k}{2}, k=\overline{0,3}\right\}$ such that on the same connected component of the curve (3.9) there are two different points $\left(r_{1}, \varphi^{*}\right)$ and $\left(r_{2}, \varphi^{*}\right), r_{1} \neq r_{2}$, lying on the ray $\varphi=\varphi^{*}$. Then from (3.9) considering (3.12) we obtain the identity equality

$$
\begin{gathered}
\cos \left(\varphi^{*}\right)\left(\left.\frac{\partial R\left(r \cos \left(\varphi^{*}\right)\right)}{\partial r}\right|_{r=r_{1}}-\left.\frac{\partial R\left(r \cos \left(\varphi^{*}\right)\right)}{\partial r}\right|_{r=r_{2}}\right) \\
=\sin \left(\varphi^{*}\right)\left(\left.\left(\frac{1}{r} \frac{\partial R\left(r \cos \left(\varphi^{*}\right)\right)}{\partial r}\right)\right|_{r=r_{1}}-\left.\left(\frac{1}{r} \frac{\partial R\left(r \cos \left(\varphi^{*}\right)\right)}{\partial r}\right)\right|_{r=r_{1}}\right),
\end{gathered}
$$


from which after the replacement $x=r \cos \left(\varphi^{*}\right)$ follows

$$
\left.\frac{d R(x)}{d x}\right|_{x_{1}=r_{1} \cos \left(\varphi^{*}\right)}=\left.\frac{d R(x)}{d x}\right|_{x_{2}=r_{2} \cos \left(\varphi^{*}\right)} .
$$

Consequently, in order to the curve (3.9) on each of its connected component determine $r$ as a single-valued function of the argument $\varphi$, some condition/requirement is necessary under which the identity equality (3.13) will not take place

$$
\left.\frac{d R(x)}{d x}\right|_{x_{1}=r_{1} \cos \left(\varphi^{*}\right)} \neq\left.\frac{d R(x)}{d x}\right|_{x_{2}=r_{2} \cos \left(\varphi^{*}\right)} .
$$

A sufficient condition/requirement of such kind is, for example, the requirement of strict monotonicity of the conductivity function $\frac{d R(x)}{d x}$. However, it must be emphasized that this requirement (i.e. strict monotonicity of $\frac{d R(x)}{d x}$ ) is not the unique condition sufficient for the single-value property of the function. One can find weaker quantitative and/or qualitative ones instead of this requirement for $\frac{d R(x)}{d x}$.

Now, let us try to find out the nature of the connected components of the curve (3.9). To do this, we return to the equation (3.10), the solution of which is

$$
y_{1,2}=\frac{-L x \frac{d R(x)}{d x} \pm \sqrt{\left(L x \frac{d R(x)}{d x}\right)^{2}-4 L C x^{2}}}{2 L C} .
$$

Four cases are possible here:

$$
\begin{aligned}
& \left\{x \geq 0 ; \frac{d R(x)}{d x} \in\left[2 \sqrt{\frac{C}{L}},+\infty\right)\right\} ; \\
& \left\{x \geq 0 ; \frac{d R(x)}{d x} \in\left(-\infty,-2 \sqrt{\frac{C}{L}}\right]\right\} ; \\
& \left\{x \leq 0 ; \frac{d R(x)}{d x} \in\left[2 \sqrt{\frac{C}{L}},+\infty\right)\right\} ; \\
& \left\{x \leq 0 ; \frac{d R(x)}{d x} \in\left(-\infty,-2 \sqrt{\frac{C}{L}}\right]\right\}
\end{aligned}
$$

Before studying these cases, we note that at the "finite boundary points" $x=0$ and/or $\frac{d R(x)}{d x}= \pm 2 \sqrt{\frac{C}{L}}$ of these cases takes place $y_{1}=y_{2}=\mp \frac{x}{\sqrt{L C}}$. Therefore we consider these cases at "internal points".

Consider the first case when $x>0$ and $\frac{d R(x)}{d x} \in\left(2 \sqrt{\frac{C}{L}},+\infty\right)$. For this case we have $y_{1}>-\frac{x}{\sqrt{L C}}$ and $y_{2}<-\frac{x}{\sqrt{L C}}$, which are obtained using the following two chains of simple arithmetic operations, respectively:

$$
\frac{d R(x)}{d x}>2 \sqrt{\frac{C}{L}} \Leftrightarrow-4 \sqrt{\frac{L}{C}} \frac{d R(x)}{d x}<-8 \Leftrightarrow
$$




$$
\begin{aligned}
& \sqrt{L C}\left(\sqrt{\frac{L}{C}} \frac{d R(x)}{d x}-2\right) x<\sqrt{L C} x \sqrt{\left(\sqrt{\frac{L}{C}} \frac{d R(x)}{d x}\right)^{2}-4}, \forall x>0 \Leftrightarrow \\
& \underbrace{\frac{L x \frac{d R(x)}{d x}+\sqrt{\left(L x \frac{d R(x)}{d x}\right)^{2}-4 L C x^{2}}}{2 L C}}_{y_{1}}>\frac{x}{\sqrt{L C}} \text {; } \\
& \frac{d R(x)}{d x}>2 \sqrt{\frac{C}{L}} \Leftrightarrow
\end{aligned}
$$

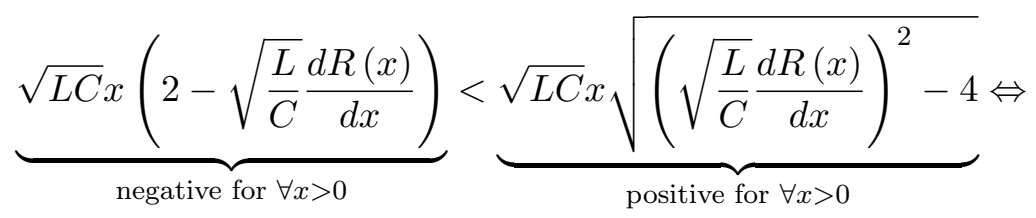

$$
\begin{aligned}
& \underbrace{\frac{L x \frac{d R(x)}{d x}-\sqrt{\left(L x \frac{d R(x)}{d x}\right)^{2}-4 L C x^{2}}}{2 L C}}_{y_{2}}<-\frac{x}{\sqrt{L C}} \text {. }
\end{aligned}
$$

For the second case when $x>0$ and $\frac{d R(x)}{d x} \in\left(-\infty,-2 \sqrt{\frac{C}{L}}\right]$ from the expression for $y_{1}$ immediately follows that $y_{1}>\frac{x}{\sqrt{L C}}$. Using the following chain of the simple arithmetic operations, we obtain the estimate $y_{2}<\frac{x}{\sqrt{L C}}$ :

$$
\begin{aligned}
& \frac{d R(x)}{d x}<-2 \sqrt{\frac{C}{L}} \Leftrightarrow \\
& \left(L \frac{d R(x)}{d x}\right)^{2}+4 L \sqrt{L C} \frac{d R(x)}{d x}+4 L C<\left(L \frac{d R(x)}{d x}\right)^{2}-4 L C \Leftrightarrow \\
& \Rightarrow \underbrace{\sqrt{\left(L \frac{d R(x)}{d x}+2 \sqrt{L C}\right)^{2}}}<\sqrt{\left(L \frac{d R(x)}{d x}\right)^{2}-4 L C} \\
& =-\underbrace{\left(L \frac{d R(x)}{d x}+2 \sqrt{L C}\right)}_{\text {negative }} \\
& -x\left(L \frac{d R(x)}{d x}+2 \sqrt{L C}\right)<x\left(\sqrt{\left(L \frac{d R(x)}{d x}\right)^{2}-4 L C}\right), \forall x>0 \Leftrightarrow \\
& \underbrace{\frac{L x \frac{d R(x)}{d x}-\sqrt{\left(L x \frac{d R(x)}{d x}\right)^{2}-4 L C x^{2}}}{2 L C}}_{y_{2}}<\frac{x}{\sqrt{L C}} .
\end{aligned}
$$

Consideration of the cases

$$
\left\{x<0 ; \frac{d R(x)}{d x} \in\left(2 \sqrt{\frac{C}{L}},+\infty\right)\right\}
$$


and

$$
\left\{x<0 ; \frac{d R(x)}{d x} \in\left(-\infty,-2 \sqrt{\frac{C}{L}}\right)\right\}
$$

gives us exactly the same results as the results of the first two cases considered above: if $\left\{x<0 ; \frac{d R(x)}{d x} \in\left(2 \sqrt{\frac{C}{L}},+\infty\right)\right\}$ then $y_{1}>-\frac{x}{\sqrt{L C}}$ and $y_{2}<-\frac{x}{\sqrt{L C}}$; if $\left\{x<0 ; \frac{d R(x)}{d x} \in\left(-\infty,-2 \sqrt{\frac{C}{L}}\right)\right\}$ then $y_{1}>\frac{x}{\sqrt{L C}}$ and $y_{2}<\frac{x}{\sqrt{L C}}$.

Putting together the results obtained regarding all four cases, we can write: - if $x=0$ and/or $\frac{d R(x)}{d x}= \pm 2 \sqrt{\frac{C}{L}}$, then $y_{1}=y_{2}=\mp \frac{x}{\sqrt{L C}}$ takes place; - if $\frac{d R(x)}{d x} \in\left(2 \sqrt{\frac{C}{L}},+\infty\right)$ and if $x>0$ or $x<0$, then the inequalities $y_{1}>$ $-\frac{x}{\sqrt{L C}}$ and $y_{2}<-\frac{x}{\sqrt{L C}}$ are take place the sense of which is quite obvious: straight lines, which are parallel to the abscissa axis, intersect the curve (3.9) at no more than two points, which are located on the opposite sides of the ray $y=-\frac{x}{\sqrt{L C}}$ of the quadrantal angles II and IV;

- if $\frac{d R(x)}{d x} \in\left(-\infty,-2 \sqrt{\frac{C}{L}}\right)$ and if $x>0$ or $x<0$, then the inequalities $y_{1}>\frac{x}{\sqrt{L C}}$ and $y_{2}<\frac{x}{\sqrt{L C}}$ are take place the sense of which is quite obvious: straight lines, which are parallel to the abscissa axis, intersect the curve (3.9) at no more than two points, which are located on the opposite sides of the ray $y=\frac{x}{\sqrt{L C}}$ of the quadrantal angles I and III.

So, the results obtained allow us to formulate the following theorem.

Theorem 3.1. (I) If the current-voltage characteristic $R(x)$ of the nonlinear resistive element of the Biryukov damped oscillator (2.1) (regardless of the fulfilment or non-fulfilment of the condition (2.2)) is such that the corresponding reduced equation (3.1) is not the Riccati equation (i.e. $\frac{d r}{d \varphi}=\frac{r P_{1}(r, \varphi)}{P_{2}(r, \varphi)} \neq$ $U_{1}(\varphi) r+U_{2}(\varphi) \frac{1}{r}+U_{3}(\varphi)$, where $U_{j}(\varphi), j=\overline{1,3}$ are some functions of an argument $\varphi$ ), then the limit cycles of the Biryukov equation (2.1) (with or without condition (2.2)), if any, represent an algebraic curve on the coordinate plane $x O \dot{x}$.

(II) If the current-voltage characteristic $R(x)$ of the nonlinear resistive element of the Biryukov damped oscillator (2.1) (with condition (2.2)) is such that the values range of the conductivity function $\frac{d R(x)}{d x}$ is the interval $\left(-2 \sqrt{\frac{C}{L}}, 2 \sqrt{\frac{C}{L}}\right)$, then the curve $\tilde{P}_{2}(r, \varphi) \stackrel{\text { def }}{\equiv} \frac{1}{r} \sin (\varphi) P_{2}(r, \varphi)=0$, where $P_{2}(r, \varphi)$ is a denominator of the right-hand side of the reduced equation (3.1), has no connected component; if the values range of the conductivity function is the region $\left(-\infty,-2 \sqrt{\frac{C}{L}}\right] \cup$ $\left[2 \sqrt{\frac{C}{L}},+\infty\right)$, on the left ray of which the conductivity function $\frac{d R(x)}{d x}$ strictly decreases, and in the right ray - strictly increases, then the curve $\tilde{P}_{2}(r, \varphi)=0$ has connected components having the following properties: firstly, on each connected 
component, a function $r(\varphi):\left\{\left(0, \frac{\pi}{2}\right) \cup\left(\frac{\pi}{2}, \pi\right) \cup\left(\pi, \frac{3 \pi}{2}\right) \cup\left(\frac{3 \pi}{2}, 2 \pi\right)\right\} \rightarrow(0,+\infty)$ is a one-valued function; secondly,

- at $\frac{d R(x)}{d x}= \pm 2 \sqrt{\frac{C}{L}}$ there is $x(t)=A e^{\mp \frac{t}{\sqrt{L C}}}, \forall A \equiv$ const, i.e. $\dot{x} \pm \frac{x}{\sqrt{L C}}=$ 0 ;

- at $\frac{d R(x)}{d x} \in\left(-\infty,-2 \sqrt{\frac{C}{L}}\right)$ and $x \in(0,+\infty)$ (or at $x \in(-\infty, 0)$ ), straight lines $x=A \equiv$ const and the curve $\tilde{P}_{2}(r, \varphi)=0$ cannot intersect at more than two points, and these points can only located on the opposite sides of the ray $\dot{x}=\frac{x}{\sqrt{L C}}$ of the coordinate angle $I$ (respectively, of the coordinate angle III for $x \in(-\infty, 0))$ of the plane $x O \dot{x}$, i.e. there are $x_{1}(t)>A_{1} e^{\frac{t}{\sqrt{L C}}}$ and $x_{2}(t)<A_{2} e^{\frac{t}{\sqrt{L C}}}$, where $A_{1}$ and $A_{2}$ are arbitrary constants.

- at $\frac{d R(x)}{d x} \in\left(2 \sqrt{\frac{C}{L}},+\infty\right)$ and $x \in(0,+\infty)$ (or at $x \in(-\infty, 0)$ ), straight lines $x=A \equiv$ const and the curve $\tilde{P}_{2}(r, \varphi)=0$ cannot intersect at more than two points, and these points can only located on the opposite sides of the ray $\dot{x}=-\frac{x}{\sqrt{L C}}$ of coordinate angle $I V$ (respectively, coordinate angle II for $x \in(-\infty, 0)$ ) of the plane $x O \dot{x}$, i.e. there are $x_{1}(t)>A_{1} e^{-\frac{t}{\sqrt{L C}}}$ and $x_{2}(t)<A_{2} e^{-\frac{t}{\sqrt{L C}}}$, where $A_{1}$ and $A_{2}$ are arbitrary constants;

Now we give two real physical examples that illustrate part (II) of the Theorem 3.1 (i.e. when the curve $\tilde{P}_{2}(r, \varphi)$ has no connected components).

Example 1. Let us consider the system

$$
\left\{\begin{array}{l}
\ddot{x}-\omega_{B} \dot{y}=0, \\
\ddot{y}+\omega_{B} \dot{x}=\omega_{E},
\end{array}\right.
$$

which describes (in a first-order approximation) in $\mathbb{R}^{3}$ motion of charged particles with mass $m$ and charge $q$ in a crossed electromagnetic field, where the electric field $\vec{E}$ directed along the axis $\overrightarrow{O Y}$ (i.e. $\left.\vec{E}=(0, E, 0)^{T}, \operatorname{dim} E=V \cdot m^{-1}\right)$ is oriented perpendicularly to the magnetic field $\vec{B}$ directed along the axis $\overrightarrow{O Z}$ (i.e. $\left.\vec{B}=(0,0, B)^{T}, \operatorname{dim} B=T\right)$.

In $(3.14), \vartheta_{0}$ is the initial velocity of the particle (i.e. $\vec{\vartheta}(t)=\left(\vartheta_{0}, 0,0\right)^{T}$, $\operatorname{dim} \vartheta_{0} \sim m \cdot s^{-1}$ ) which is considered to be constant and to lie in the $X O Y$ plane (obviously, in this case particles' trajectories also lie in the $X O Y$ plane); $\omega_{E}=\frac{q E}{m} ; \omega_{B}=\frac{q B}{m}$.

It is easy to verify that after transition to a new coordinate system moving with some constant speed $\vartheta_{\text {new }}$ relative to the original coordinate system the system (3.14) transforms into

$$
\left\{\begin{array}{l}
\ddot{x}-\omega_{B} \dot{y}=0, \\
\ddot{y}+\omega_{B} \dot{x}=\omega_{E}-\omega_{B} \vartheta_{n e w},
\end{array}\right.
$$

which, setting $\vartheta_{\text {new }}=\frac{\omega_{E}}{\omega_{B}}$, easily transforms into the Biryukov equation (2.1) (condition (2.2) is satisfied) with $\omega_{B}=\frac{1}{\sqrt{L C}}, R(x)=-\frac{t}{L} x(t)+\tilde{R}(t)$, where $\tilde{R}(t)$ is any function independent of $x$, for example, $\tilde{R}(t) \equiv 0$. Under initial conditions $x(0+0)=0, \dot{x}(0+0)=\vartheta_{0}, y(0+0)=0, \dot{y}(0+0)=0$ the following 
functions are the solution of (3.14): $x(t)=-\frac{\vartheta_{\text {new }}-\vartheta_{0}}{\omega_{B}} \sin \left(\omega_{B} t\right)+\vartheta_{\text {new }} t, y(t)=$ $\frac{\vartheta_{n e w}-\vartheta_{0}}{\omega_{B}}\left(1-\cos \left(\omega_{B} t\right)\right)$.

In Fig. 4 one can see dynamics of trajectory of the above-described charged particles on the phase plane (implemented in Mathcad, version 14.0.0.163).

Example 2. Now, consider another simple example - dynamics of a nonharmonic oscillator of arbitrary amplitude (not necessarily small oscillations) with damping $\lambda$ and restoring force $\sin (\omega x(t))$. Then, denoting by $x(t)$ the angle of deviation of the oscillator from the vertical axis $\overrightarrow{O Z}$, we will get the equation $\ddot{x}-\lambda \dot{x}+\omega^{2} \sin (\omega x)=0$, which, obviously, is a special case of the classic Liénard equation (1.1) with $f(x)=-\lambda \equiv$ const and $g(x)=\omega^{2} \sin (\omega x)$. In Fig. 5 one can see integral curves of the considered nonharmonic oscillator with damping values $\lambda$ going from 0 to 5 with a step of 1 (implemented in Mathcad, version 14.0.0.163).

\section{Briefly on the announced in the abstract a series of works on fractional order Liénard type equations planned by the authors}

As it was mentioned in the Section 2 of the present paper, the Biryukov equation (2.1) (all the more the Liénard equation) cannot be solved by analytical methods, and existing numerical methods have significantly different accuracy, stability, convergence rate, and complexity. Therefore, the question arises of choosing a more efficient numerical method. However, evaluating the effectiveness of a concrete numerical method is an independent non-trivial problem that requires complex additional theoretical studies, and a comparative analysis of the effectiveness of several numerical methods for solving (2.1) is an even more complex problem.

Incommensurably more complex difficulties have to be dealt with when studying nonlinear differential equations with fractional order derivatives, in particular, Liénard-type equations with fractional derivatives. Currently, such kind of equations are quite relevant in connection with their widespread use as mathematical models for studying various processes with anomalous kinetics (for instance, see [38], [54]). It should be emphasized here that, unlike the classical derivative of an integer order, for differential equations with fractional order derivatives there are many non-identical definitions (for instance, see [48], [31], [40], [27], [32], [29], [20]), and these non-identical definitions generate classes of differential equations with fractional order derivatives, in particular, of equations of Liénardtype, which are close in form, but are significantly different in properties. Most often, in practice, the concepts of left-side fractional derivatives of order $\alpha>0$ in the sense of Riemann-Liouville (for instance, see [48])

$$
{ }_{c}^{R L} D_{t}^{\alpha} x \stackrel{\text { def }}{\equiv} \frac{1}{\Gamma(n-\alpha)} \frac{d^{n}}{d t^{n}} \int_{c}^{t} \frac{x(\tau) d \tau}{(t-\tau)^{\alpha-n+1}},
$$


in the sense of Caputo (for instance, see [9] as well as [31])

$$
{ }_{c}^{C} D_{t}^{\alpha} x \stackrel{\text { def }}{\equiv} \frac{1}{\Gamma(n-\alpha)} \int_{c}^{t} \frac{x^{(n)}(\tau) d \tau}{(t-\tau)^{\alpha-n+1}},
$$

or in the sense of Hadamard (for instance, see [22] as well as [48])

$$
{ }_{c}^{H} D_{t}^{\alpha} x \stackrel{\text { def }}{\equiv} \frac{\alpha}{\Gamma(n-\alpha)} \int_{c}^{t}\left(\log \frac{t}{\tau}\right)^{n-\alpha-1} \frac{x(\tau)}{\tau} d \tau
$$

are used. Here $n=[\alpha]+1$ if $\alpha \notin \mathbb{N}$, and $n=\alpha$ if $\alpha \in \mathbb{N} ; \Gamma(\ldots)$ is the gamma function that is known as the Euler integral of the second kind: $\Gamma(z)=$ $\int_{0}^{\infty} \tau^{z-1} e^{-\tau} d \tau, \operatorname{Re}(z)>0$.

In the general case, the solution of a differential equation with fractional order derivative in the sense of Riemann-Liouville can contain an integrable singularity of order at most $(1-\alpha)$ at the point $t=c$, while the existence of derivative in the sense of Riemann-Liouville implies the boundedness of the solution at this point. It is known (for instance, see [31]) that if there exists a finite limit $\lim _{t \rightarrow c+0} x(t)=x(c)$, then derivatives in the sense of Riemann-Liouville and in the sense of Caputo are related by

$$
{ }_{c}^{R L} D_{t}^{\alpha} x={ }_{c}^{C} D_{t}^{\alpha} x+\frac{1}{\Gamma(1-\alpha)} \frac{x(c)}{(t-c)^{\alpha}} .
$$

In the work [6] some well-known methods for constructing point groups of transformations were modified and evolved for differential equations with fractional order derivatives, understood both in the sense of Riemann-Liouville and in the sense of Caputo.

It is important to note that all definitions including foregoing satisfy the property that the fractional derivative is linear. This is the only property inherited from the first derivative by all of the definitions: many of these definitions do not satisfy the well-known rules and properties of derivatives of the natural order, such as the derivative of the product, the derivative of the quotient, the chain rule, the semigroup property, etc. A successful attempt to eliminate these drawbacks is the concept of a local fractional derivative, which arose only in 2014 in [29], when the definition of "conformable fractional derivative" was introduced, which is defined in terms of a certain quotient incremental, in consequence of which a local fractional derivative appears: the "conformable fractional derivative" of a function $x(t)$ of order $\alpha \in(0,1)$ is the operator $T_{\alpha}$ defined by

$$
T_{\alpha} x(t) \stackrel{\text { def }}{=} \lim _{\varepsilon \rightarrow 0} \frac{x\left(t+\varepsilon t^{1-\alpha}\right)-x(t)}{\varepsilon}, \forall t \in \mathbb{R}_{++} .
$$

In 2018 by the works [20], [41] a local fractional derivative of a new type, "nonconformable fractional derivative", was introduced. Exactly within the scope of this concept, the next work of the authors will devoted to the study of some qualitative properties of the solutions of one hybrid differential-fractional system of Lienar type obtained from the classical model (see [14], [21], [42]). 


\section{Conclusion}

In the present paper, the behaviour of limit cycles of the Biryukov equation (Biryukov oscillator) is investigated. A quantitative condition (in the form of equality) with respect to the current-voltage characteristic of nonlinear resistive element of the Biryukov damped oscillator is found, upon fulfilment of which the closed trajectory of the equation is an algebraic curve, otherwise, either all solutions of Biryukov equation are periodic or the number of periodic solutions, if any, is not more than two. In other words, it is shown that either all solutions of the Biryukov equation are closed or the Biryukov equation can have no more than two limit cycles. In addition, in this paper, we found a simple sufficient condition of a qualitative nature with respect to the conductivity function of the Biryukov oscillator, the fulfilment of which ensures the uniqueness of the solution of the Biryukov equation: such a sufficient condition is the condition of strict monotonicity of the current-voltage characteristic of the nonlinear resistive element of the Biryukov damped oscillator outside a certain interval, the boundaries of which also identified.

\section{Acknowledgements}

For the first two co-authors the present paper is executed within the framework of the Investment and Development Agency of Latvia (www.liaa.gov.lv/lv) project No. KC-PI-2020/36 titled "Investigation and Development of Self-cleaning Hydrophobic and Hydrophilic Nano-coatings for Photovoltaic Converters and Solar Thermal Collectors".

The second author of the present paper would like to express his deepest gratitude and appreciation to Professor Mansur I. Ismailov from the Department of Mathematics, Faculty of Science, Gebze Technical University, Gebze, Turkey, for his kind help in providing some hard-to-reach materials on the present research issue.

\section{References}

[1] V. I. Arnold, V. S. Afraimovich, Yu. S. Ilyashenko, L. P. Shilnikov, Bifurcation theory, "Results of Science and Technology", Vol. 5: "Dynamical Systems" Moscow, USSR: VINITI (All-Union Institute for Scientific and Technical Information), 1986, $5-218$.

[2] M. A. Barron, Stability of a ring of coupled van der Pol oscillators with non-uniform distribution of the coupling parameter, Journal of Applied Research and Technology, 14(2016), no.1, 62-66.

[3] M. A. Barron, M. Sen, Synchronization of four coupled van der Pol oscillators, Nonlinear Dynamics, 56(2009), no.4, 357-367.

[4] P. J. Beek, R. C. Schmidt, A. W. Morris, M. Y. Sim, M. T. Turvey, Linear and nonlinear stiffness and friction in biological rhythmic movements, Biological Cybernetics, 73(1995), no.6, 499-507.

[5] V. N. Biryukov, L. E. Gatko, Exact stationary solution of the oscillator nonlinear differential equation, Nonlinear World, 10(2012), no.9, 613-616.

[6] E. Buckwar, Yu. Luchko, Invariance of a partial differential equation of fractional order under the Lie group of scaling transformations, Journal of Mathematical Analysis and Applications, 227(1998), no.1, 81-97. 
[7] J. C. Butcher, The Numerical Analysis of Ordinary Differential Equations: RungeKutta and General Linear Methods, Chichester, UK: John Wiley \& Sons, 1987.

[8] J. C. Butcher, Numerical Methods for Ordinary Differential Equations, Chichester, UK: John Wiley \& Sons, 2003.

[9] M. Caputo, Lineal model of dissipation whose Q is almost frequency independent-II Geophysical Journal International, 13(1967), no.5, 529-539.

[10] Sh. H. Chang, I. L. Chang, A new algorithm for calculating one-dimensional differential transform of nonlinear functions, Applied Mathematics and Computation, 195(2008), no.2, 799-808.

[11] T. L. Chow, Classical Mechanics, Boca Raton, USA: CRC Press, 2013.

[12] M. S. Dutra, A. C. de Pina Filho, V. F. Romano, Modelling of a bipedal locomotor using coupled nonlinear oscillators of Van der Pol, Biological Cybernetics, 88(2003), no.4, 286-292.

[13] A. E. H. Ebaid, Approximate periodic solutions for the non-linear relativistic harmonic oscillator via differential transformation method, Communications in Nonlinear Science and Numerical Simulation, 15(2010), no.7, 1921-1927.

[14] A. Fleitas, J. A. Mendez-Bermudez, J. E. Napoles Valdes, J. M. Sigarreta Almira, On fractional Lienard-type systems, Revista Mexicana de Física, 65(2019), no.6, 618-625.

[15] G. R. Fowles, G. L. Cassiday, Analytic Mechanics, Belmont, USA: Thomson Brooks/Cole, 2005.

[16] V. V. Golubev, Lectures on the Analytical Theory of Differential Equations, MoscowLeningrad, USSR: State Publishing House of Technical and Theoretical Literature, 1950 .

[17] D. Greenspan, Particle Modelling, Boston, USA: Birkhäuser Basel, 1997.

[18] S. N. Gurbatov, O V Rudenko, A I Saichev, Waves and Structures in Nonlinear Nondispersive Media: General Theory and Applications to Nonlinear Acoustics, Berlin, Germany: Springer, 2011.

[19] D. S. Guzhev, N. N. Kalitkin, Burgers equation is a test for numerical methods, Mathematical Modelling, 7(1995), no.4, 99-127.

[20] P. M. Guzman, G. Langton, L. M. Lugo, J. Medina, J. E. Napoles Valdes, A new definition of a fractional derivative of local type, Journal of Mathematical Analysis, $\mathbf{9}(2018)$, no.2, 88-98.

[21] P. M. Guzman, J. E. Napoles Valdes, A note on the oscillatory character of some non-conformable generalized Lienard system, Advanced Mathematical Models 83 Applications, 4(2019), no.2, 127-133.

[22] L. Hadamar, Essai sur l'étude des fonctions données par leur développement de Taylor, Journal de mathématiques pures et appliquées, 4e série, VIII, (1892), 101186.

[23] E. Hairer, S. P. Norsett, G. Wanner, Solving Ordinary Differential Equations. Volume I: Nonstiff Problems, Berlin, Germany: Springer-Verlag, 2008.

[24] E. Hairer, G. Wanner, Solving Ordinary Differential Equations. Volume II: Stiff and Differential-Algebraic Problems, Berlin, Germany: Springer-Verlag, 2010.

[25] Yu. S. Ilyashenko, An example of eqations $d w / d z=\operatorname{Pn}(\mathrm{z}, \mathrm{w}) / \mathrm{Qn}(\mathrm{z}, \mathrm{w})$ having a countable number of limit cycles and arbitrarily large Petrovskii-Landis genus, Mathematical Collection (Sbornik Mathematics), 80(1969), no.3, 388-404.

[26] D. W. Jordan, P. Smith, Nonlinear Ordinary Differential Equations: Problems and Solutions, Oxford, UK: Oxford University Press, 2007.

[27] G. Jumarie, Modified Riemann-Liouville derivative and fractional Taylor series of non-differentiable functions further results, Computers \& Mathematics with Applications, 51(2006), no.9-10, 1367-1376. 
[28] T. Kawahara, Coupled Van der Pol oscillators - A model of excitatory and inhibitory neural interactions, Biological Cybernetics, 39(1980), no.1, 37-43.

[29] R. Khalil, N. Al Horani, A. Yousef, M. Sababheh, A new definition of fractional derivative, Journal of Computational and Applied Mathematics, 264(2014), 65-70.

[30] M. G. Khudai-Verenov, A remark on limit cycles of an equation, Differential Equations, 3(1967), no.4, 692-694.

[31] A. A. Kilbas, H. M. Srivastava, J. J. Trujillo, Theory and Applications of Fractional Differential Equations, Amsterdam, The Netherlands: Elsevier Science, 2006.

[32] K. M. Kolwankar, A. D. Gangal, Hölder exponents of irregular signals and local fractional derivatives, Pramana - Journal of Physics, 48(1997), no.1, 49-68.

[33] J. D. Lambert, Numerical Methods for Ordinary Differential Systems, Chichester, UK: John Wiley \& Sons, 2008.

[34] E. M. Landis, I. G. Petrovskii, On the number of limit cycles of the equation $\mathrm{dy} / \mathrm{dx}=\mathrm{P}(\mathrm{x}, \mathrm{y}) / \mathrm{Q}(\mathrm{x}, \mathrm{y})$, where $\mathrm{P}$ and $\mathrm{Q}$ are polynomials of 2nd degree, Mathematical Collection (Sbornik Mathematics), 37(1955), no.2, 209-259.

[35] E. M. Landis, I. G. Petrovskii, On the number of limit cycles of the equation $\mathrm{dy} / \mathrm{dx}=\mathrm{P}(\mathrm{x}, \mathrm{y}) / \mathrm{Q}(\mathrm{x}, \mathrm{y})$, where $\mathrm{P}$ and $\mathrm{Q}$ are polynomials, Mathematical Collection (Sbornik Mathematics), 43(1957), no.2, 149-168.

[36] E. M. Landis, I. G. Petrovskii, Letter to the Editors (On error in the proof of the Lemma 12 in the article "On the number of limit cycles of the equations $\mathrm{dy} / \mathrm{dx}=\mathrm{P}(\mathrm{x}, \mathrm{y}) / \mathrm{Q}(\mathrm{x}, \mathrm{y})$, where $\mathrm{P}$ and $\mathrm{Q}$ are polynomials of 2nd degree", Mathematical Collection (Sbornik Mathematics), 73(1967), 160.

[37] D. A. Linkens, I. Taylor, H. L. Duthie, Mathematical modelling of the colorectal myoelectrical activity in humans, IEEE Transactions on Biomedical Engineering, 23(1976), no.2, 101-110.

[38] R. Metzler, J. Klafter, The random walk's guide to anomalous diffusion: A fractional dynamic approach, Physics Reports, 339(2000), no.1, 1-77.

[39] R. E. Mickens, Periodic solutions of the relativistic harmonic oscillator, Journal of Sound and Vibration, 212(1998), no.5, 905-908.

[40] A. M. Nakhushev, Fractional Calculus and its Application, Moscow, Russia: FizMatLit Publishing House, 2003.

[41] J. E. Napoles Valdes, P. M. Guzman, L. M. Lugo, Some new results on nonconformable fractional calculus, Advances in Dynamical Systems and Applications, 13(2018), no.2, 167-175.

[42] J. E. Napoles Valdes, P. M. Guzman, L. M. Lugo, A. Kashuri, The local non-conformable derivative and Mittag-Leffler function, ResearchGate,

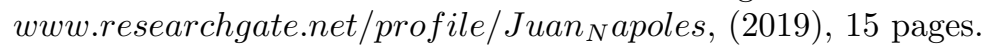

[43] N. F. Otrokov, On the number of limit cycles of a differential equation in the neighbourhood of a singular point, Mathematical Collection (Sbornik Mathematics), 34(1954), no.1, 127-144.

[44] I. G. Petrovskii, E. M. Landis, Corrections to the articles "On the number of limit cycles of the equations $\mathrm{dy} / \mathrm{dx}=\mathrm{P}(\mathrm{x}, \mathrm{y}) / \mathrm{Q}(\mathrm{x}, \mathrm{y})$, where $P$ and $Q$ are polynomials of 2nd degree" and "On the number of limit cycles of the equation $d y d x=P(x, y) Q(x, y)$, where P and Q are polynomials, Mathematical Collection (Sbornik Mathematics), 48(1959), no.2, 253-255.

[45] A. M. Pilipenko, V. H. Biryukov, Investigation of modern numerical analysis methods of self-oscillatory circuits' efficiency, Journal of Radio Electronics, (2013), no.8.

[46] V. P. Popov, Fundamentals of Circuit Theory, Moscow, USSR: "Higher School" Publishing, 1985.

[47] P. L. Sachdev, On linear Ordinary Differential Equations and their Applications, New York, USA: Marcel Dekker, 1991. 
[48] S. G. Samko, A. A. Kilbas, O. I. Matichev, Fractional Integrals and Derivatives, and Some of Their Applications, Minsk, USSR: Science and Engineering, 1987.

[49] H. G. Schuster, W. Just, Deterministic Chaos: An Introduction Schuster, Weinheim, Germany: Wiley-VCH Verlag, 2005.

[50] P. Smith, Mechanics, Chichester, UK: John Wiley \& Sons, 1990.

[51] I. Stewart, D. Tall, Complex Analysis, Cambridge, UK: Cambridge University Press, 1983.

[52] J. W. Strutt, Third Baron Rayleigh, The Theory of Sound, Cambridge, UK: Cambridge University Press, 2011.

[53] J. M. T. Thompson, H. B. Stewart, Nonlinear Dynamics and Chaos, West Sussex, UK: John Wiley \& Sons, 2002.

[54] V. V. Uchaykin, Method of Fractional Derivatives, Ulyanovsk, Russia: "Artishok" Publishings, 2008.

[55] K. Weierstrass, Uber die analytische Darstellbarkeit sogenannter willkürlicher Functionen einer reellen Veränderlichen, Sitzungsberichte der Akademie zu Berlin (1885), 789-805.

Yusif S. Gasimov

Azerbaijan University, 71 J.Hajibeyli Street, Baku AZ1007, Azerbaijan.

Institute of Mathematics and Mechanics, Azerbaijan National Academy of Sciences, 9 B. Vahabzade Street, Baku AZ1148, Azerbaijan.

Institute of Physics Problems, Baku State University, 23 Z.Khalilov Street, Baku AZ1148, Azerbaijan.

E-mail address: gasimov ·yusif@gmail.com

Sharif E. Guseynov

Faculty of Science and Engineering, Liepaja University, 14 Liela Street, Liepaja $L V$-3401, Latvia.

Institute of Fundamental Science and Innovative Technologies, 14 Liela Street, Liepaja LV-3401, Latvia.

"Entelgine" Research 8 Advisory Co., Ltd., 2 Kleistu Street, Riga LV-1067, Latvia.

E-mail address: sh.e.guseinov@inbox.lv

Juan E. Nápoles Valdés

FUNNE, FaCENA, Ave. Libertad 5450, Corrientes 3400, Argentina.

UTN-FRRE, French 414, Resistencia, Chaco 3500, Argentina.

E-mail address: jnapoles@exa.unne.edu.ar

Received: April 27, 2020; Revised: October 9, 2020; Accepted: October 23, 2020 


\section{ORIGIN: $=1$}

Limit_cycles := for $n \in 1.20$

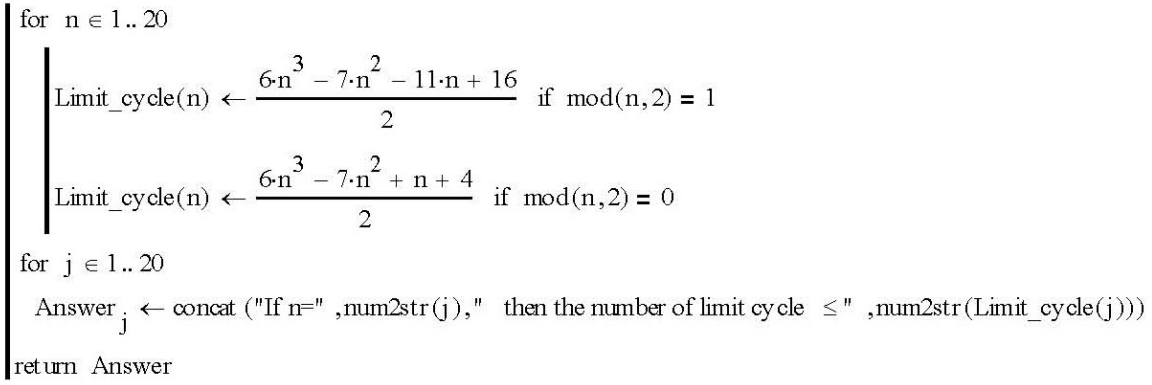

Maximum_possible_number_of_limit_cycles := Limit_cycles

Maximum_possible_number_of_limit_cycles

FiguRE 1. Listing of MathCad 14 Program realizing the upper bound of the number of limit cycles of the equation (3.5). 


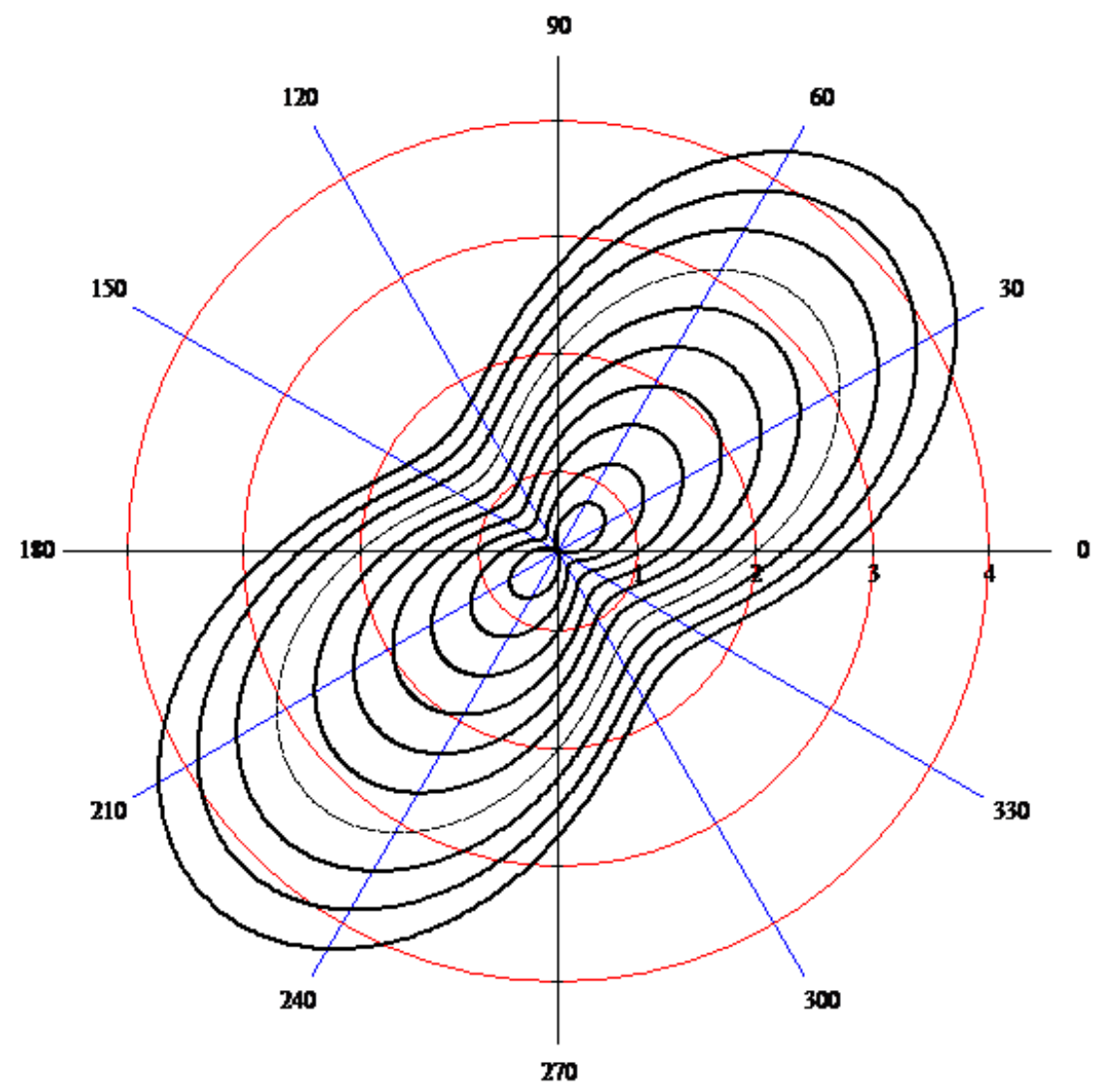

FiguRE 2. Integral curves of the first reduced equation. 


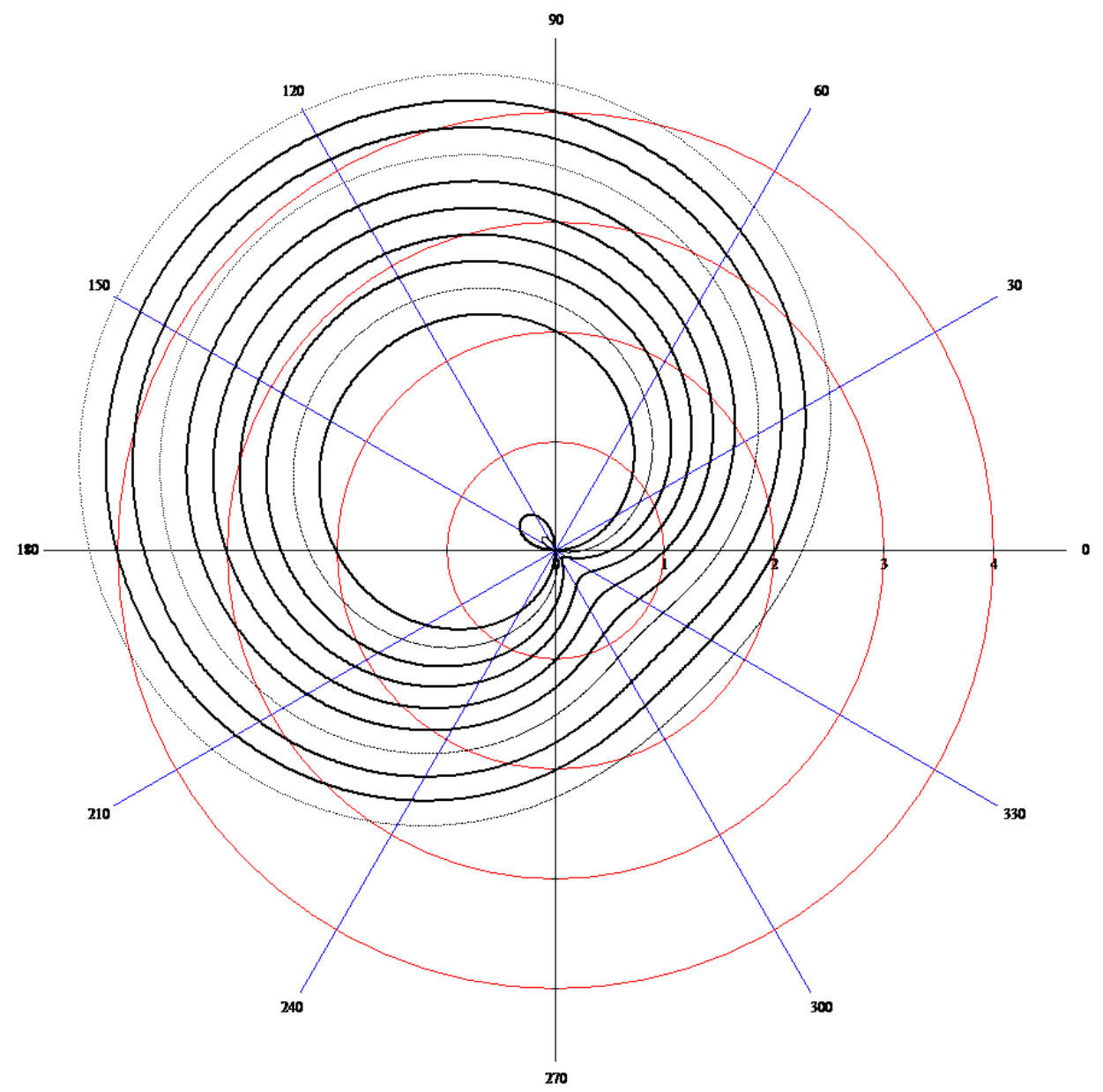

FiguRE 3. Integral curves of the second reduced equation. 


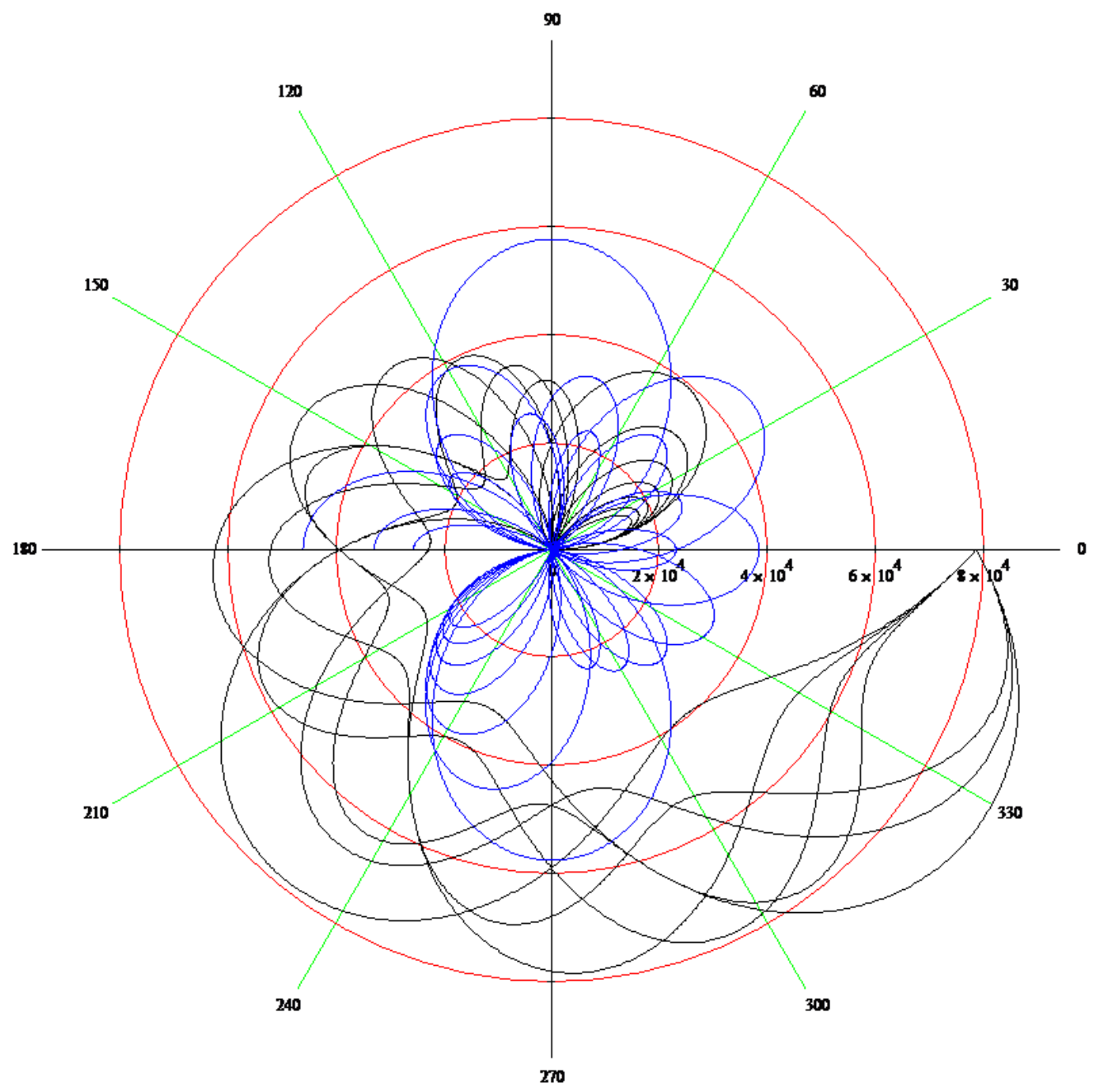

FiguRE 4. The phase-plane portrait of the dynamics of the trajectory of charged particles with m-mass and q-charging in a crossed electromagnetic field: $\mathrm{x}(\mathrm{t})$ is shown in black, and $\mathrm{y}(\mathrm{t})$ is shown in blue. 


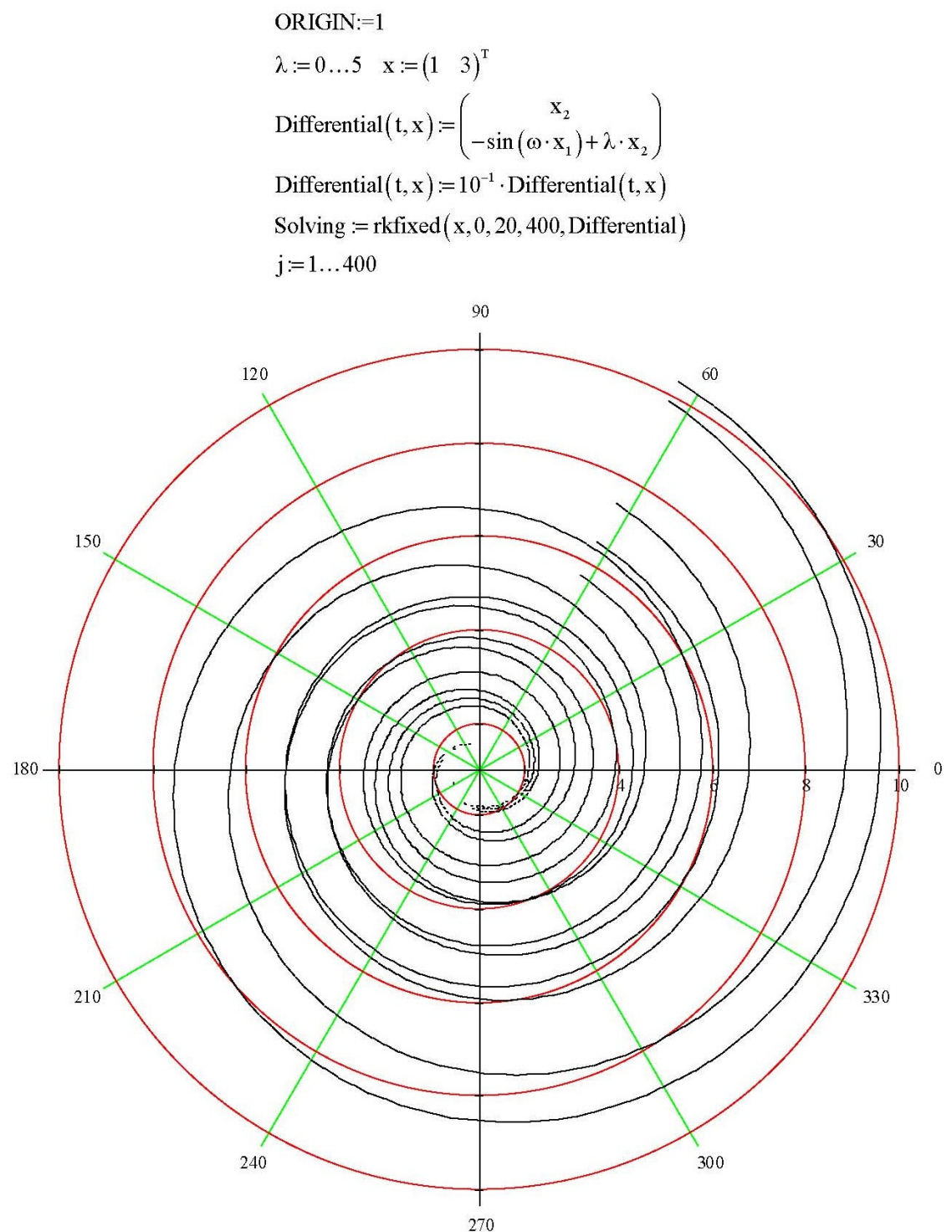

FiguRE 5. Integral curves of the nonharmonic oscillator from the illustrative Example 2 (realization listing in Mathcad is included also). 